\title{
Estudio de un cemento bioactivo de hidroxiapatita como material de substitución ósea
}

\author{
J.J. MORALES DE CANO*, A. GARCÍA RUZAFA*, J.A. PLANELL ESTANY**, M.P. GINEBRA MOLINS**, \\ M.DRIESSENS** Y J.A. HERNÁNDEZ HERMOSO*. \\ *Servicio de Cirugía Ortopédica y Traumatología. \\ Ciudad Sanitaria y Universitaria de Bellvitge. Barcelona. \\ ** Departamento de Ciencia de los Materiales e Ingeniería Metalurgica.
}

ETS Ingenieros Industriales

\begin{abstract}
Resumen
La cirugía ortopédica y máxilo-facial necesita realizar en muchas ocasiones resecciones masivas de tejido óseo. Esto ha hecho que se haya propuesto la utilización de materiales inertes como substitutos óseos, gracias a la habilidad que tienen de permitir la regeneración del hueso, tanto en el campo de la medicina como en el de la odontología. Nuestro trabajo tiene por objetivo estudiar un biomaterial compuesto de hidroxiapatita y dicalcio-fosfato (hidroxiapatita deficiente en calcio) como material de substitución ósea, en forma de cemento bioactivo.

Se han intervenido 75 animales de experimentación (conejo Albino de Nueva Zelanda), divididos en dos grupos, cada uno de ellos compuesto por 25 animales. En el grupo I o control, los animales fueron intervenidos quirúrgicamente realizándoseles una cavidad a nivel metáfisodiafisario en el fémur sin realizar implantes. A los animales del grupo II se les realizó la misma intervención, pero se les implantó un cilindro fraguado de hidroxiapatita deficiente en calcio.
\end{abstract}

Palabras clave: Biomateriales, hidroxiapatita, cemento bioactivo, osteoconducción, substitutos óseos.

\section{Introducción}

En los últimos años, se ha introducido la utilización de biomateriales como tratamiento de los defectos óseos en la cirugía ortopédica y máxilo-facial. Los carbonatos cálcicos son conocidos por su buena biocompatibilidad, pero su obtención y procesamiento, así como sus propiedades intrínsecas han hecho que se relegue su utilización a un segundo plano. El sulfato cálcico, biomaterial bien tolerado, no citotóxico, plantea problemas en su utilización aislada debido a su alta solubilidad y rápida

\section{Correspondencia:}

J.J. Morales de Cano

La plana $\mathrm{n}^{\circ}$ 15. San Cugat del Vallés.

08190 Barcelona

Spain reabsorción por los fluidos corporales. La hidroxiapatita (HA) muestra una satisfactoria biocompatibilidad y osteoconducción, lo que ha provocado que sea uno de los biomateriales más investigado y utilizado como substituto óseo. Dentro del subgrupo de los fosfatos cálcicos se han introducido otros compuestos como el tricalcio fosfato, dicalcio y octofosfato cálcicos, entre otros.

La utilización de los biomateriales ha ido incorporando nuevas formas de presentación, adecuándose a las necesidades de la cirugía y al mejor aprovechamiento de las propiedades físicoquímicas de dichos materiales. Es de todos conocida la utilización de biomateriales en forma de polvo con mayor o menor tamaño de gránulo. También se han utilizado los materiales en "bloque": pastillas, cilindros, etc. Más recientemente se ha incorporado la filosofía de utilizar materiales en forma de pasta, que tras un corto tiempo de fraguado se solidifiquen, éstos son los llamados cementos 
En el presente trabajo, realizamos un estudio de experimentación, en el que se estudia un cemento bioactivo compuesto de fosfato tricálcio $\alpha$ e hidroxiapatita precipitada.

\section{Material y métodos}

\section{Material de implante}

Utilizamos como material a estudio la composición:

- 98\% fosfato tricálcio $\alpha: \alpha-\mathrm{Ca}_{3}\left(\mathrm{PO}_{4}\right)_{2}$

- $2 \%$ hidroxiapatita precipitada

- Líquido de disolución: solución 2.5\% $\mathrm{Na}_{2} \mathrm{HPO}_{4}$

La relación entre el líquido de disolución y los compuestos es de 0.32 .

El producto de reacción una vez fraguados los compuestos es la hidroxiapatita deficiente en calcio.

Para la elaboración de los implantes se utilizó la mezcla descrita y se introdujo la pasta formada en un molde cilíndrico de teflón con un tamaño de 11 $\mathrm{mm}$ de longitud, por $6 \mathrm{~mm}$ de diámetro y se introdujo en una estufa durante un tiempo de 24 horas a temperatura ambiente.

Las propiedades mecánicas del material de implante son:

- Tiempo inicial de fraguado del cemento bioactivo: 7.5 minutos

- Tiempo final de fraguado: $15 \mathrm{~min}$.

- Resistencia mecánica a la compresión de los cilindros: $40 \mathrm{MPa}$. (se alcanza a las $24 \mathrm{~h}$. Del fraguado)

El material fue envasado en viales, precintados y esterilizados con rayos gamma a una dosis de 25 KGray, en el Centro Aragogamma ( $\left.n^{\circ} 1\right)$.

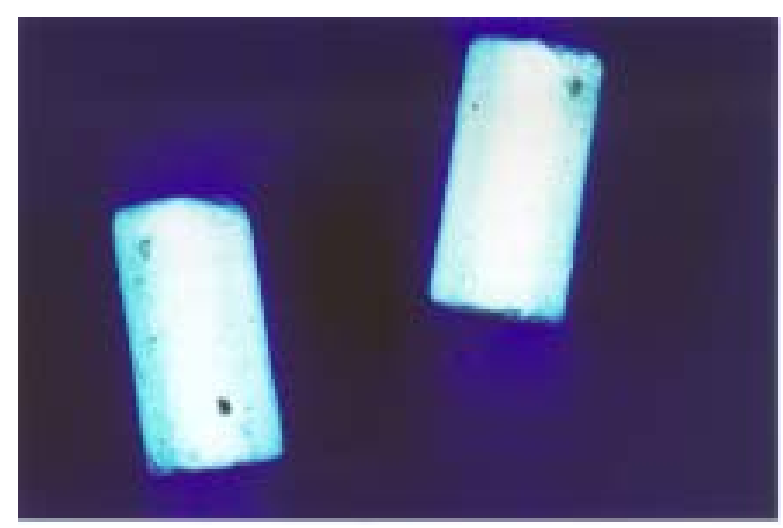

Figura 1: Cilindros de hidroxiapatita deficiente en calcio.

\section{Animal de experimentación}

El estudio se realizó en el conejo albino de Nueva Zelanda, todos machos y adultos con edades comprendidas entre 4 y 5 meses, con un peso de $3,5-4 \mathrm{Kg}$. Los animales fueron cuidados en el estabulario con las normas habituales (temperatura de la habitación de $20^{\circ} \mathrm{C}$, humedad relativa de $55.5 \%$, cambio del aire 20 veces / hora).

En total hemos intervenido 40 animales de experimentación, divididos en dos grupos:

1. Animales del grupo I o grupo control, constituido por 20 animales. Estos animales fueron intervenidos realizándoles una cavidad metáfisodiafisaria distal en el fémur, sin colocación de implante.

2. Animales del grupo II, constituido por 20 animales. Éstos han sido intervenidos de igual manera que los del grupo control, pero en este caso se les implantó un cilindro de hidroxiapatita deficiente en calcio (cemento óseo ya fraguado).

\section{Técnica quirúrgica}

Todos los animales fueron intervenidos bajo anestesia general endovenosa con una mezcla de ketamina y xilacina. Se realizó una incisión medial a nivel del tercio distal femoral con abordaje subvasto medialis de la zona metáfiso-diafisaria del fémur mediante una broca de $6 \mathrm{~mm}$. Estas cavidades fueron rellenadas mediante el material sujeto de estudio (Figura $\mathrm{n}^{\circ} 2$ ). Al finalizar la intervención quirúrgica la extremidad intervenida de los animales fue inmovilizada mediante un vendaje de yeso pelvipédico.

Los animales fueron sacrificados a tiempos diferentes: entre 3 días y 5 semanas los animales pertenecientes al grupo I, y entre 3 días y 6 semanas los animales del grupo II.

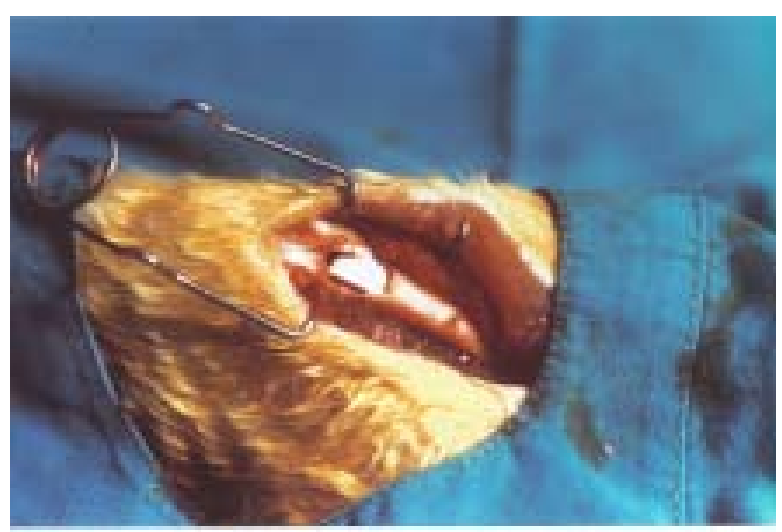

Figura 2: Implantación del material en el fémur del animal de experimentación. 


\section{Estudio postoperatorios}

Desde el momento de la intervención y hasta el sacrificio de los animales se realizó el seguimiento de los siguientes parámetros:

1. Determinaciones bioquímicas:

- Calcemia.

- Fosfatemia.

- Determinación de fosfatasas alcalinas séricas.

Estas determinaciones se realizaron preoperatorias, a las dos y seis semanas.

2. Estudio radiográfico: se realizaron controles radiográficos cada quince días desde el momento de la intervención hasta el sacrificio de los animales.

3. Estudio histológico: al producirse el sacrificio de los animales. Se realizó mediante:

- Estudio histológico descriptivo con microscopía óptica de hueso descalcificado y sin decalcificar.

- Estudio histomorfométrico analizando:

- Superficie de material implantado.

- Superficie de hueso formado.

Tratamiento estadístico de los resultados

Los datos obtenidos en los resultados fueron tratados estadísticamente mediante las pruebas de Chicuadrado, t de Student y prueba de Wilcoxon, fijándose la significación estadística en $\mathrm{p}<0.05$.

\section{Resultados}

\section{Determinaciones bioquimicas}

Los resultados de las determinaciones de calcio en suero nos dieron en los animales del grupo I cifras comprendidas entre 13.98 y $15.30 \mathrm{mg} /$ dl. En los animales del grupo II los valores obtenidos oscilaron entre 11.70 y $14.90 \mathrm{mg} / \mathrm{dl}$. Todos los resultados nos muestran valores dentro de la normalidad. El tratamiento estadístico de estas determinaciones entre los animales del grupo I y II fue de $p>0.05$, por consecuencia, sin diferencia estadísticamente significativa.

En cuanto a la cuantificación del fosfato inorgánico en plasma, los valores oscilaron entre 4.35 y $7.65 \mathrm{mg} / \mathrm{dl}$ en el grupo control, y sin diferencias con las determinaciones de los conejos del grupo II.

La determinación de las fosfatasas alcalinas en los animales del grupo I nos dieron cifras comprendidas entre 49 y $306 \mathrm{U} / \mathrm{l}$. Por su parte las determinaciones en los animales del grupo II nos dieron cifras entre 72 y $337 \mathrm{U} / 1$ (Grafica $\mathrm{n}^{\circ} 1$ ).

Los resultados de las determinaciones analíticas demuestran que los animales del grupo II presentan unos valores similares a los del grupo control, sin diferencias estadísticas.

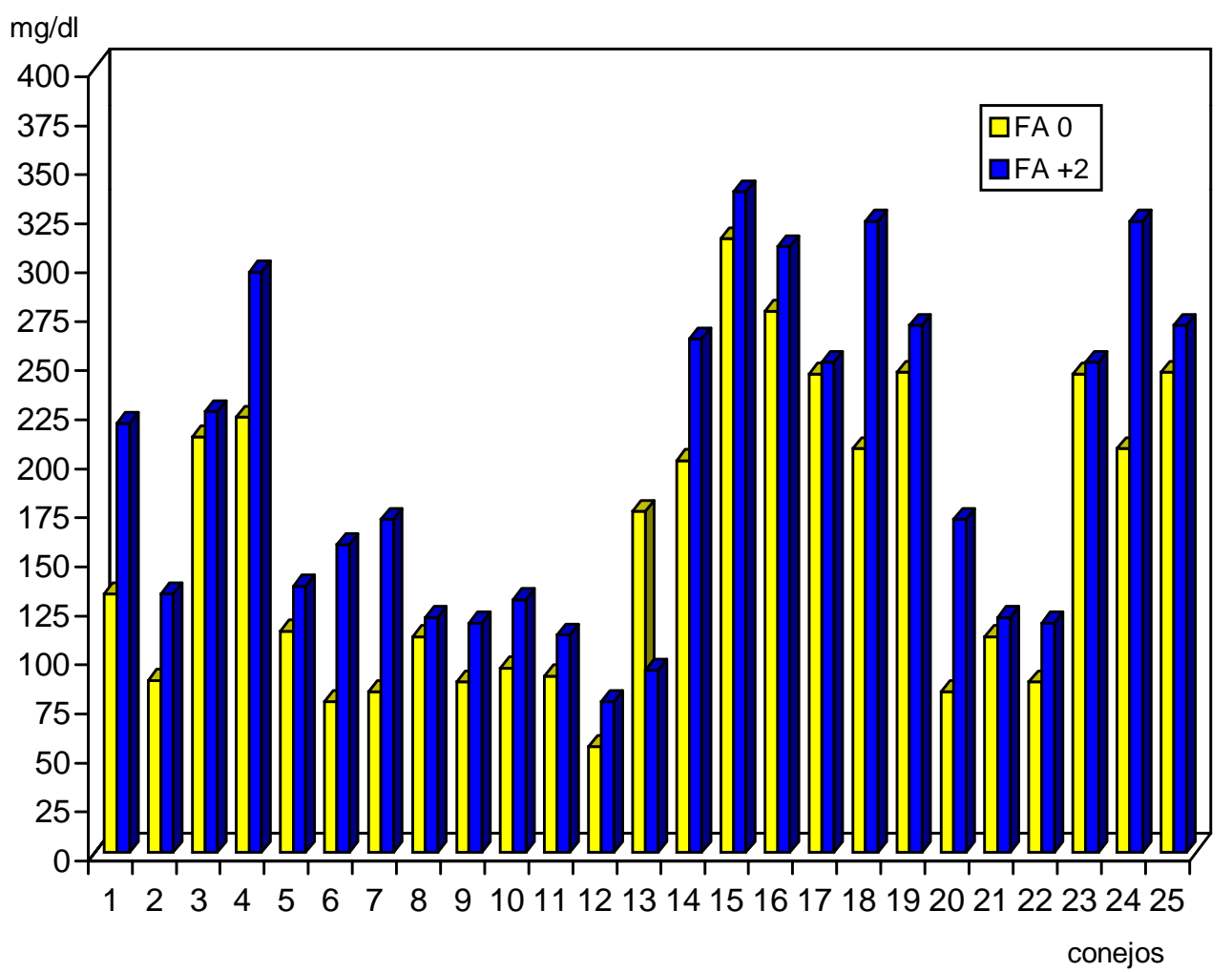

Gráfico 1: Comparación de las determinaciones de fosfatasa alcalina sérica entre los animales del grupo control y los del grupo II, a las seis semanas. 


\section{Estudio radiográfico}

Las radiografías realizadas en el postoperatorio inmediato a los animales intervenidos nos muestran la zona metáfiso-diafisaria del hueso donde ha sido implantado el material con un aumento de la densidad ósea debida al aspecto radiopaco del material implantado (Figura $n^{\circ} 3$ ). En los controles radiográficos ulteriores, se observa una mínima zona de disminución de la densidad cálcica en la periferia del implante, que difumina el contorno del cilindro implantado (Figura nº ${ }^{\circ}$ ). En ningún momento hemos podido constatar una fragmentación del material. Al final del estudio, los cilindros permanecen casi intactos, sin producirse reabsorción del material a estudio.

\section{Estudio histológico}

En las preparaciones realizadas a los tres días después de la intervención se puede apreciar como el material implantado está rodeado de tejido hemorrágico en muy poca cantidad, favorecido por la agresión quirúrgica. A las dos semanas, en los animales del grupo II, observamos muy poco tejido infamatorio con escasa celularidad. Aparecen algunos macrófagos y casi nula reacción de cuerpo extraño. El hallazgo de células gigantes a cuerpo extraño es excepcional. Esto nos indica la buena tolerancia del animal al material implantado. En puntos muy concretos, y siempre en la periferia del implante, se aprecia la aparición de ribetes con actividad osteoblástica y hueso plexiforme neoformado, aunque en escasa cantidad (Figura $\left.\mathrm{n}^{\circ} 5\right)$.

A las seis semanas, en los animales del grupo II, se sigue apreciando una estrecha franja de tejido fibroso cicatricial que rodea al implante, y del que

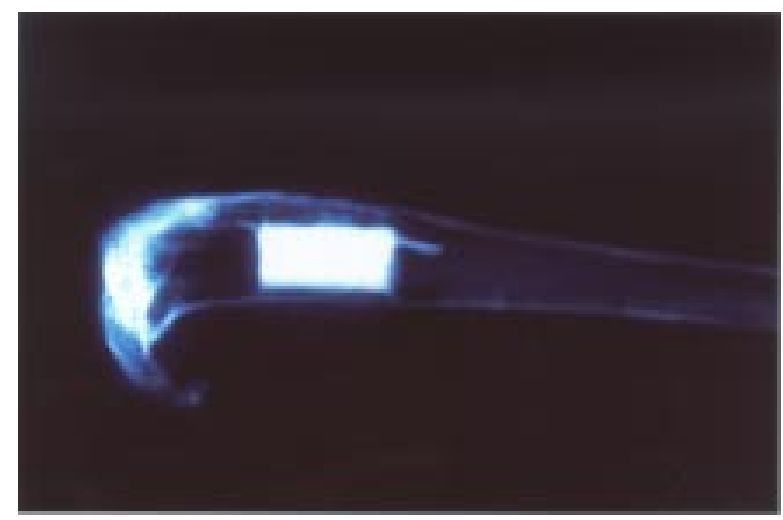

Figura 3: Radiografía postoperatoria del fémur de un animal de experimentación del grupo II. Zona metáfisodiafisaria con aumento de densidad ósea correspondiente a la zona del implante del material. emergen trabéculas óseas neoformadas, con poca actividad osteoblástica y osteoclástica (Figura nº ${ }^{\circ}$ ). No se observa proliferación vascular en la periferia del implante. El material permanece casi intacto.

El estudio histomorfométrico en los animales del grupo II demuestra una baja reabsorción del material implantado, con poca área de hueso plexiforme neoformado.

El estudio histológico muestra una buena tolerancia del material implantado sin reacción a cuerpo extraño, poca reacción inflamatoria y baja formación ósea.

\section{Discusión}

Las cerámicas de fosfatos cálcicos se consideran materiales bioactivos según la definición propuesta por Williams en 1987 (36): "Material que induce una actividad biológica especifica". Las cerámicas de fosfato cálcico actúan interaccionando con el hueso reabsorbiéndose más o menos lentamente dependiendo del tipo y de la porosidad del material. Esto ha hecho que la hidroxiapatita pueda ser considerada como un material biocompatible. Por esta razón se ha utilizado como material de relleno cavitario, o como material de recubrimiento $(1,2,4,5,18,21,25,26,27,28,29,32)$

Los estudios histológicos de los fosfatos cálcicos han demostrado que aunque no son materiales que puedan actuar como osteoinductores, sí que actúan como soporte del crecimiento óseo, lo que se ha denominado osteoconducción $(19,20,22,23,34,35,36)$. La utilización de la hidroxiapatita en forma de granos compactos con una porosidad baja presenta grandes inconvenientes ya que no permite la formación de las trabéculas

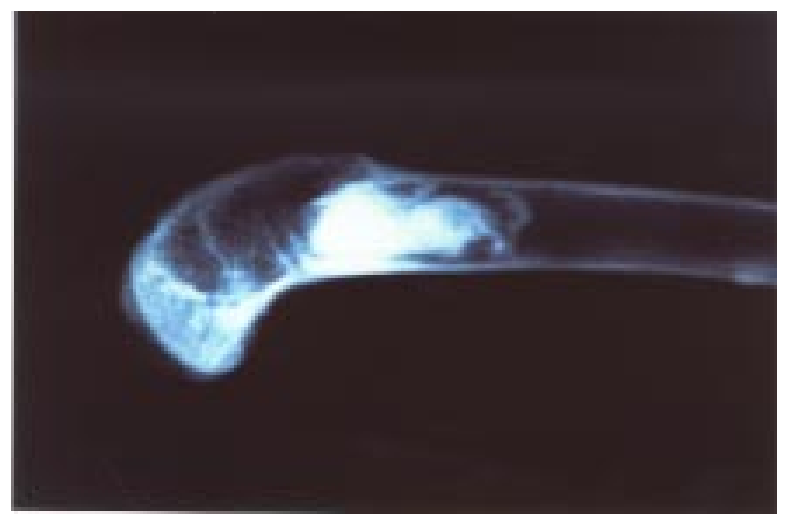

Figura 4: Radiografía a las seis semanas del implante, en un animal del grupo II. Se aprecia discreta disminución de la zona radiopaca, 
óseas recién formadas.

En nuestro trabajo, hemos confeccionado un cemento bioactivo compuesto por hidroxiapatita y dicalcio fosfato (HA deficiente en calcio) y hemos pretendido investigar su poder osteoconductor (15). Las propiedades intrínsecas del material son muy atractivas, ya que pasa de una fase semilíquida a sólida de forma rápida, cualidad muy valorada de cara al relleno de cavidades. Sin embargo, después de asimilar los resultados de nuestro estudio, creemos conveniente destacar, que con nuestro material, la actividad osteoconductiva ha sido mucho más moderada que con otros materiales sólidos de forma definitiva. Quizás sería conveniente sacrificar las propiedades y la utilidad de un cemento bioactivo para aumentar su poder osteoconductor, que en sí es nuestro objetivo final $(4,5,6,14)$

La evaluación clínica de los implantes es difícil de cuantificar, pero sin embargo los parámetros bioquímicos de calcio y fosfatasa alcalina séricos nos sirven como valoración indirecta de un aumento de la actividad osteoblástica, aunque moderada, en los animales del grupo II con respecto al grupo control.

En contra estudios realizados con HA compacta, en nuestro estudio no hemos comprobado la formación de tejido fibroso que encapsule el implante, ni tampoco se forma una reacción generalizada de cuerpo extraño.

Algunos autores han descrito alteraciones en el calcio plasmático en animales a los que se les han implantado algunos tipos de materiales (25). En nuestro estudio hemos comprobado incremento de las cifras de calcio sérico, manteniéndose dentro del rango de los valores fisiológicos. No hemos podido hallar diferencias significativas en las determinaciones de calcio, fósforo ni fosfatasas alcalinas

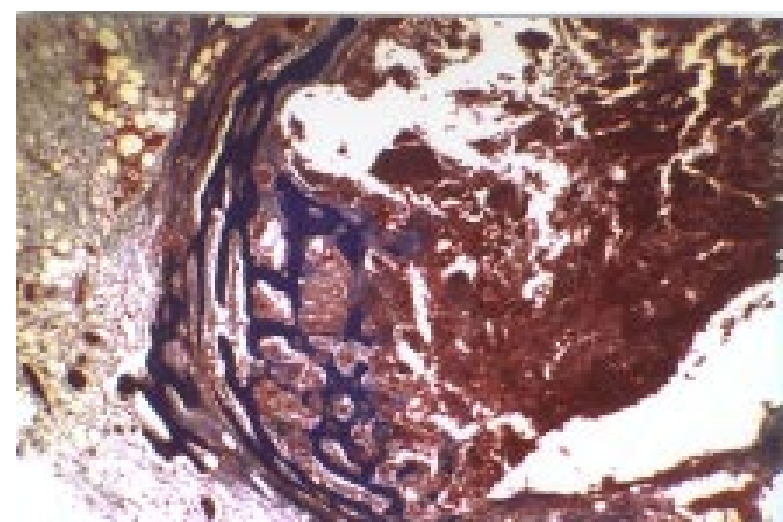

Figura 5: MO 400x. Tricrómico de Masson: A las dos semanas del implante, se observa la buena tolerancia del material implantado. en los animales de los grupos a estudio.

\section{Conclusiones}

Como resumen de nuestro trabajo podemos afirmar que el cemento bifásico formado por hidroxiapatita deficiente en calcio es un material biocompatible, sin reacción a cuerpo extraño. Posee unas propiedades muy atractivas de cara al relleno de cavidades, por el hecho de pasar de forma rápida de una fase semilíquida a sólida, pero en contra, actúa como osteoconductor de forma muy moderada.

\section{Agradecimientos}

Este estudio se ha realizado gracias a la subvención de la beca CICYT (Ref. MAT98-0976C02-02).

\section{Biblografía}

1. Beirne OR, Grenspan JS. "Histologic evaluation of tissue response to hidroxyapatite implanted on human mandibles." J. Dent. Res., 64: 1152, 1985.

2. Block MS, Kent JN, Kay JF. "Evaluation of hidroxyapatite coated titanium dental implants in dogs." J.oral Maxilofacial Surg., 45: 601, 1987.

3. Chiroff RT, White E, Roy D, Weber J. "Tissue ingrowth of replamineform implants." J. Biomed. Mater. Res., 6: 29-45, 1975.

4. Daculsi G, Legeros RZ, Mitre D. "Cristal dissolution of biological and ceramic apatites." Calcif. Tiss. Int., 1988.

5. Daculsi G, Passuti N, Martin S, Deudon C, Legeros RZ, Raher S. "Macroporous calcium phosphate ceramic for long bone surgery in humans and dogs. Clinical and histological study." J.Biomedical Mat.

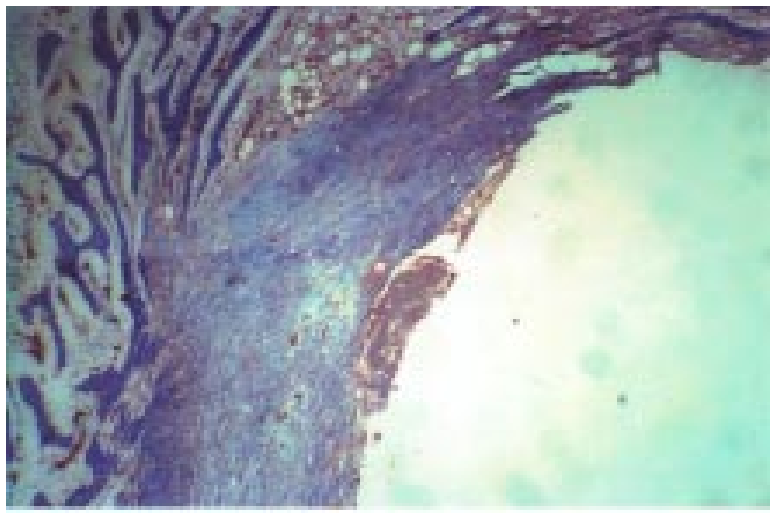

Figura 6: MO 400x. Tricrómico de Masson. Seis semanas después del implante. 
Research., 24: 379-396, 1990.

6. Doll B.A., Towle H.J., Hollinger J.O., Reddi A.H., Mellonig J.T. "The osteogenic potential of two composite graft systems using osteogenin." J. Periodontal, 61: 745-750, 1990.

7. Drobeck HP. "Histopathologic observations for long term soft tissue responses following implantation of random shaped particles and discs of durapatite." J. Oral Maxillofac. Surg., 42(3): 143149, 1984.

8. Eysel W, Roy DM. "Topotactic reaction of aragonite to hydroxyapatite." Z. Kristollographic, 141: 11-15, 1975.

9. Finn R, Brammer JA. "Interpositional "grafting" with autogenous bone and coralline hydroxyapatite." J. Maxillofac. Surg., 8: 217-227, 1980.

10. Froum S, Stahl SS. "Human intraosseus healing responses to the placement of tricalcium phosphate ceramic implants. II. 13 to 18 months." J. Periodontol., 58: 103-109, 1987.

11. Glass DA, Mellonig JT, Towle HJ. "Histologic evaluation of bone inductive proteins complexed with coralline hydroxyapatite in an extraskeletal site of the rat." J. Periodontol., 60: 121-126, 1989.

12. Han T, Carranza FA, Kenney EB. "Calcium phophate ceramics in dentistry." J. West Soc. Periodont. Abstr., 32: 88, 1984.

13. Holmes RE. "Bone regeneration within a coralline hydroxyapatite implant." Plast. Reconst. Surg., 63: 626-633, 1979.

14. Iwano T., Kurosawa H., Murase K. "Tissue reaction collagen-coated porous hydroxyapatite." Clin. Orthop., 268: 243-252, 1991.

15. Jarcho M, Bolen CH, Thomas MB, Bobick J, Kau JF, Doremus RH. "Hydroxyapatite synthesis and characterization in dense polycristaline form." J. Matr Sci., 11: 2027-2035, 1976.

16. Jarcho M. "Calcium phosphate ceramic as hard tissue prosthetics." Clin. Orthop., 157: 259-277, 1981.

17. Jarcho M, Salsbury RL, Thomas MB, Doremus RH. "Synthesis and fabrication of beta-tricalcium phosphate ceramics for potential prosthetic applications." J. Matr. Sci., 14: 142-148, 1979.

18. Kattagen B. "Bone with bone substitutes." Ed. Springer-Verlag. Berlin, 1988.

19. Kenney EB, LekovicV, Han J, Carranza FA, Dimitrejevic B. "The use of porous hydroxyapatite implants in periodontal defects." J. Periodontol., 56: 82-88, 1985.

20. Kenney EB, LekovicV, Sa Ferreira JC. Han T, Dimitrijevic B, Carranza F. "Bone formation within porous hydroxyapatite implants in human periodontal defects." J. Periodontol., 57: 76-83, 1986.

21. Kent JN, Quinn JH, Zide MF. "Alveolar rige augmentation using nonresorbable hydroxyapatite with or without autogenous cancellous bone." J.oral Maxilofac. Surg., 41: 629, 1983.
22. Klein CP, Driessen AA, de Groot $K$. "Biodegradation behaviour of various calcium phosphate materials in bone tissue". J Biomed. Mater. Res., 17: 769, 1983.

23. Meffert RM, Thomas JR, Hamilton KM. "Hydroxyapatite as an alloplastic gract in the treatment of human periodontal osseous defects." J. Periodeon., 56: 63, 1985.

24. Metsger JS, Drikell TD, Paulsrud JR. "Tricalcium phosphate ceramic, a resorbable bone implant: Review and current status." J. Am. Dent. Assoc., 105; 1035-1038, 1982.

25. Morales JJ. "Experimental del relleno de cavidades óseas con sulfato cálcico impregnado con gentamicina." Tesis Doctoral. Universidad de Barcelona. 1991.

26. Morales de Cano JJ, Peraire C, García Ruzafa A, Hachem A, Fernàndez Sabaté A. "Estudio de la citotoxicidad del sulfato cálcico como material de substitución ósea." Biomecánica, 10: 8-14, 1998.

27. Morales de Cano JJ, García Ruzafa A, Peraire C, Fernández Sabaté A. "Estudio de la biocompatibilidad del sulfato cálcico como material de substitución ósea." Avances, 28: 153-164, 1998.

28. Morales de Cano JJ, Romero RuizJ, García Ruzafa A, Fernández Sabaté A. "Estudio experimental del relleno de cavidades óseas con hidroxiapatita asociada a colágeno." Biomecánica, Vol. 9: 101-107, 1997.

29. Ohno K, Sugimoto A, Shirota T. "Histologic finding of apatite-titanium complex dental implants in the jaw of dogs." Oral Surg. Oral Med. Oral Pathol., 71: 426, 1991.

30. Redja DA. "Tricalcium phosphate as a bone substitute." J. Bioeng., 1: 93-98, 1977.

31. Redja DA. "Composite materials for hard tissue replacement" (Thesis). Amsterdam, The Netherlands: Free University, 1977.

32. Renoij W, Hoogendoorn H, Visser WJ, Lentferink RH, Scmitz MG, Van Ieperen H, Oldenburg SJ, Janssen WM, Akkermans LM, Wittebol P. "Bioresorption of ceramic strontium85 labelled calcium phosphate ceramic implants in dog femora." Clin. Orthop., 197: 272, 1985.

33. Roy DM, Linnehan S. "Hydroxyapatite formed from coral skeleton carbonate by hydrothermal exchange." Nature, 247: 220-222, 1974.

34. Urist MR, Lietze A, Dawson E. "Beta-tricalcium phosphate delivery system for bone morphogenetic protein." Clin. Orthop., 187: 277-280, 1984.

35. West TL, Brustein DD. "Comparison of replamineform coral and bone alloimplants in dog periodontal implants." J. Dent. Res., 57(Spec. Issue): $101,1978$.

36. Williams DF. "Definitions in Biomaterials. Progress in Biomedical Engineering." Ed. Elsevier, Amsterdam, 1987. 